\title{
An energy analysis of convective instabilities of the Bödewadt and Ekman boundary layers over rough surfaces
}

\author{
B. Alveroglu ${ }^{1 *}$, A. Segalini ${ }^{2}$, S.J. Garrett ${ }^{3}$
}

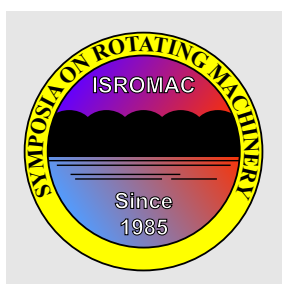

ISROMAC 2016

International

Symposium on

Transport

Phenomena and

Dynamics of

Rotating Machinery

Hawaii, Honolulu

April 10-15, 2016

\begin{abstract} presented here confirm the results of our prior linear stability analyses.

Keywords

Roughness - BEK family — Energy

*Corresponding author: ba123@le.ac.uk
${ }^{1}$ Department of Mathematics, University of Leicester, Leicester, LE1 7RH, UK
${ }^{2}$ Linné FLOW Centre, KTH Mechanics, SE-100 44, Stockholm, Sweden
${ }^{3}$ Department of Engineering, University of Leicester, Leicester, LE1 7RH, UK
\end{abstract}

An energy balance equation for the three-dimensional Bödewadt and Ekman layers of the so called "BEK family" of rotating boundary-layer flows is derived. A Chebyshev discretisation method is used to solve the equations and investigate the effect of surface roughness on the physical mechanisms of transition. All roughness types lead to a stabilization of the Type I (cross-flow) instability mode for both flows, with the exception of azimuthally-anisotropic roughness (radial grooves) within the Bödewadt layer which is destabilising. In the case of the viscous Type II instability mode, the results predict a destabilisation effect of radially-anisotropic roughness (concentric grooves) on both flows, whereas both azimuthally-anisotropic roughness and isotropic roughness have a stabilisation effect. The results

\section{INTRODUCTION}

Developing passive drag-reduction techniques has been a priority for many years. It was a classical belief that smooth surfaces are likely to generate less skin friction than rough surfaces until the recent studies have shown the opposite for some cases [1-3]. Indeed, the right sort of roughness on surfaces could lead to drag-reducing effects [2-4].

A promising strategy for drag reduction is to delay the transition of the laminar boundary-layer flow with the help of surface roughness. A recent study by Cooper et al. [4] suggests that this is possible in the case of the von Kármán [5] boundary-layer flow established over a disk rotating in otherwise still fluid. Our previous study [6] established the same drag-reducing effects for the broader BEK class of flows that includes the Bödewadt [7], Ekman [8] and von Kármán [5] boundary-layer flows. In this current study we perform an energy analysis of the Ekman and the Bödewadt flows to verify those results.

The BEK class of flows occur in turbo-machinery and rotorstator devices, such as mixers, and their stability characteristics are therefore of practical importance in engineering design applications. Many studies have been performed to reveal the similarities in the stability characteristics across the entire BEK class (see, for example, the theoretical and experimental studies discussed in papers [9-14] for the Bödewadt layer; papers $[15,16]$ for the Ekman layer; and papers [17-20] for the von Kármán flow). These studies have shown the existence of two main flow instability mechanisms, commonly referred to as the Type I and the Type II modes. The Type I mode is inviscid in nature and results from an inflectional cross-flow component of the steady flow; the Type II mode is viscous in nature and results from streamwise curvature and Coriolis effects. The instability nature of a particular flow in the BEK system is determined by the dominant one of those instability modes.

Two distinct theoretical models for the steady boundarylayer flow over rough rotating disks have been established in the literature. They were developed by Miklavčič \& Wang [21] and Yoon et al. [22], henceforth referred to as the MW and YHP models, respectively. The MW model uses a partial-slip condition on the disk surface and is capable of modelling independent levels of roughness in both the radial and azimuthal directions. The MW model can therefore model all variations of anisotropic roughness (radial and azimuthal) and also isotropic roughness. In contrast, however, the YHP approach imposes a particular surface geometry on the lower disk and can model only radial anisotropic roughness. The limitations of the YHP model motivate the use of the MW model alone in this study and our previous linear stability analysis [6].

This paper proceeds as follows: The mean-flow equations and calculations of the steady boundary-layer flows of the BEK system are summarized in Section 1. We then derive the relevant equations from the perturbed system and conduct 
energy analyses of the Ekman and the Bödewadt flows for particular disturbance eigenmodes in Section 2. Conclusions are then drawn in Section 3.

\section{THE STEADY MEAN FLOWS}

The steady-flow formulation of the BEK system for smooth surfaces is constructed in the related papers of Lingwood [17] and Lingwood \& Garrett [23]. Full details of the scalings can be found there. The formulation for rough surfaces under the MW model is detailed in our previous study [6] and we discuss it only briefly here.

The Bödewadt and the Ekman boundary-layers are two distinct cases of a general class of fully three-dimensional boundary layers within the BEK family. Within this family flows are distinguished by a differential rotation rate between a lower disk and the fluid above generated by an additional rotating disk in the far-field. The system is assumed to rotate within an otherwise still, incompressible fluid. Both disks are assumed to be of infinite extent and rotate around a common axis with angular velocities $\Omega_{D}^{*}$ (lower disk) and $\Omega_{F}^{*}$ (far field). The Ekman flow is the case that $\Omega_{D}^{*} \approx \Omega_{F}^{*}$ and the Bödewadt flow has $\Omega_{D}^{*}=0$ and $\Omega_{F}^{*} \neq 0$. Although not considered here, the von Kármán flow has $\Omega_{D}^{*} \neq 0$ and $\Omega_{F}^{*}=0$. Using a rotating frame leads to the appearance of Coriolis terms and centrifugal effects in the governing equations. Therefore, the flows in the system can be classified by the Rossby number that is a constant of the flow relating to the ratio of Coriolis forces to inertial forces. It can be formulated as

$$
R o=\frac{\Omega_{F}^{*}-\Omega_{D}^{*}}{\Omega^{*}},
$$

where the system rotation rate $\Omega^{*}$ defined as

$$
\begin{aligned}
\Omega^{*} & =\frac{\Omega_{F}^{*}}{2-R o}+\frac{\Omega_{D}^{*}}{2-R o} \\
& =\frac{\Omega_{F}^{*}+\Omega_{D}^{*}}{4}+\left(\left(\frac{\Omega_{F}^{*}+\Omega_{D}^{*}}{4}\right)^{2}+\frac{\left(\Omega_{F}^{*}-\Omega_{D}^{*}\right)^{2}}{2}\right)^{1 / 2} .
\end{aligned}
$$

In this study, we focus only on the Bödewadt $(R o=1)$ and the Ekman $(R o=0)$ boundary layers in the BEK system.

A cylindrical polar coordinate system that rotates with the lower disk is used in the formulation of the governing equations for the steady flow. The MW model is used to model the steady-boundary layer flow over a rough lower disk. The approach modifies the no-slip condition as stated below at the disk surface, $z=0$, whereas the far-field boundary conditions at the upper edge of the boundary layer are identical to the smooth-disk formulation.

$$
\begin{array}{lll}
U(0)=\lambda U^{\prime}(0), & V(0)=\eta V^{\prime}(0) & \text { and } W(0)=0, \\
U=0, & V=1, & \text { as } z \longrightarrow \infty .
\end{array}
$$

Here, $U, V$, and $W$ are the radial, azimuthal and vertical mean velocity profiles and the two parameters $\eta$ and $\lambda$ are empirical measures of roughness in radial and azimuthal directions, respectively.

The computed mean-flow profiles for different levels of surface roughness are presented in our previous study [6]. A detailed discussion about the effects of roughness on the profiles can also be found in that paper. In this study, we proceed to perform an energy analysis on the computed meanflow profiles.

\section{ENERGY ANALYSIS}

A local linear stability analysis of the BEK system with surface roughness was conducted by Alveroglu et al. [6]. Following the work of Cooper \& Carpenter [24] and Cooper et al. [4], an integral energy equation for three-dimensional disturbances $(\hat{u}, \hat{v}, \hat{w}, \hat{p})$ to the undisturbed steady mean-flow profiles $(U, V, W)$ is derived in cylindrical polar coordinate system in rotating frame to analyse the underlying physical mechanisms behind Alveroglu et al.'s conclusions. The derivation of the governing energy equations begins by multiplying the linearized momentum equations by $\hat{u}, \hat{v}$ and $\hat{w}$, respectively. Summing the resulting expressions leads to the kinetic-energy equation for the disturbances,

$$
\begin{aligned}
& \left\{\frac{\partial}{\partial t}+U \frac{\partial}{\partial r}+\frac{V}{\operatorname{Re}} \frac{\partial}{\partial \theta}-\frac{R o W}{\operatorname{Re}} \frac{\partial}{\partial z}\right\} K= \\
& -\hat{u} \hat{w} \frac{\partial U}{\partial z}-\hat{v} \hat{w} \frac{\partial V}{\partial z}+\frac{R o}{R e} \hat{w}^{2} \frac{\partial W}{\partial z}+\frac{R o U}{R e} \hat{u}^{2} \\
& +\frac{\operatorname{RoU}}{\operatorname{Re}} \hat{v}^{2}-\left[\frac{\partial(\hat{u} \hat{p})}{\partial r}+\frac{1}{\operatorname{Re}} \frac{\partial(\hat{v} \hat{p})}{\partial \theta}+\frac{\partial(\hat{w} \hat{p})}{\partial z}-\frac{\operatorname{Ro}}{\operatorname{Re} \hat{u} \hat{p}]}\right. \\
& +\left[\frac{\partial\left(\hat{u}_{j} \sigma_{i j}\right)}{\partial x_{i}}-\sigma_{i j} \frac{\partial \hat{u}_{j}}{\partial x_{i}}\right]
\end{aligned}
$$

Here $K=\frac{1}{2}\left(\hat{u}^{2}+\hat{v}^{2}+\hat{w}^{2}\right)$ and $\sigma_{i j}$ are anti-symmetric viscous stress terms. Furthermore, $R e$ is a local Reynolds number and it is written in terms of local radial position $r_{a}$ as $R e=r_{a} R o$. All $O(1 / r)$ viscous terms are now neglected to ensure consistency with the linearized governing stability equations.

We now average the perturbations over a single time period and azimuthal mode and integrate across the boundary layer. The steady, rotationally-symmetric nature of the energy is 
enforced by neglecting both $t$ and $\theta$, leading to

$$
\begin{aligned}
& \int_{0}^{\infty}[\underbrace{U \frac{\partial \bar{K}}{\partial r}}_{\mathrm{a}}+\underbrace{\frac{\partial(\overline{\hat{u} \hat{p}})}{\partial r}}_{\mathrm{b}}-\underbrace{\frac{\partial}{\partial r}\left(\overline{\hat{u} \sigma_{11}}+\overline{\hat{v} \sigma_{12}}+\overline{\hat{w} \sigma_{13}}\right)}_{\mathrm{c}}] d z \\
& =\underbrace{\int_{0}^{\infty}\left[\left(-\overline{\hat{u}} \hat{w} \frac{\partial U}{\partial z}\right)+\left(-\overline{\hat{v}} \hat{w} \frac{\partial V}{\partial z}\right)+\left(\overline{\hat{w}^{2}} \frac{R o}{\operatorname{Re}} \frac{\partial W}{\partial z}\right)\right] d z}_{\mathrm{I}} \\
& -\underbrace{\int_{0}^{\infty}\left(\overline{\sigma_{i j} \frac{\partial \hat{u}_{j}}{\partial x_{i}}}\right) d z}_{\mathrm{II}}+\underbrace{\int_{0}^{\infty}\left(\frac{R o}{\operatorname{Re}} \overline{\hat{u} \hat{p}}\right) d z+(\overline{\hat{w} \hat{p}})_{\mathrm{W}}}_{\mathrm{III}} \\
& -\underbrace{\left[\overline{\hat{u} \sigma_{31}}+\overline{\hat{v} \sigma_{32}}+\overline{\hat{w} \sigma_{33}}\right]_{\mathrm{W}}}_{\mathrm{IV}} \\
& +\underbrace{\int_{0}^{\infty} \frac{\operatorname{Ro}}{\operatorname{Re}} \frac{\partial \bar{K}}{\partial z} W d z+\int_{0}^{\infty} \frac{\operatorname{Ro}}{\operatorname{Re}} \hat{\hat{u}}^{2} U d z+\int_{0}^{\infty} \frac{\operatorname{Ro}}{\operatorname{Re}} \hat{v}^{2} U d z}_{\mathrm{V}}
\end{aligned}
$$

Overbars in the equations denote a period-averaged quantity, such that $\overline{\hat{u} \hat{v}}=\hat{u} \hat{v}^{*}+\hat{u}^{*} \hat{v}$ (where ${ }^{*}$ indicates a complex conjugate). When $R o=-1$, this equation is consistent with the energy equation for the von Kármán boundary layer [4, 24]. We now proceed with a normal-mode analysis and impose perturbations of the form

$$
(\hat{u}, \hat{v}, \hat{w}, \hat{p})=(u(z), v(z), w(z), p(z)) e^{i(\alpha r+\beta \operatorname{Re} \theta-\omega t)} .
$$

Here the radial wavenumber $\alpha=\alpha_{r}+i \alpha_{i} \in \mathbb{C}$ and the azimuthal wavenumber and frequency $\beta, \omega \in \mathbb{R}$. It is assumed that $\beta$ is $O(1)$ and the number of spiral vortices around the disk surface is given by $n=\beta R e$. Furthermore, the orientation angle of the vortices with respect to a circle centred on the axis of rotation is defined as $\epsilon=\tan ^{-1}\left(\beta / \alpha_{r}\right)$. In all that follows we set the frequency $\omega=0$ as we are interested in stationary vortices rotating with the rough disk.

As discussed elsewhere [24], the energy balance can be performed for any eigenmode of the perturbation equations and is derived after inserting (4) into (3) as

$$
\begin{aligned}
-2 \alpha_{i}= & \underbrace{\left(P_{1}+P_{2}+P_{3}\right)}_{\mathrm{I}}+\underbrace{D}_{\mathrm{II}}+\underbrace{\left(P W_{1}+P W_{2}\right)}_{\mathrm{III}} \\
& \underbrace{\left(S_{1}+S_{2}+S_{3}\right)}_{\mathrm{IV}}+\underbrace{\left(G_{1}+G_{2}+G_{3}\right)}_{\mathrm{V}},
\end{aligned}
$$

where the terms can be interpreted physically as

(I) the Reynolds stress energy production,

(II) the viscous dissipation energy removal term,

(III) pressure work terms,

(IV) contributions from work done on the wall by viscous stresses,

(V) terms arising from the streamline curvature effects and the three dimensionality of the mean flow.

The positive terms contribute to energy production and the negative ones remove energy from the system. The mode is

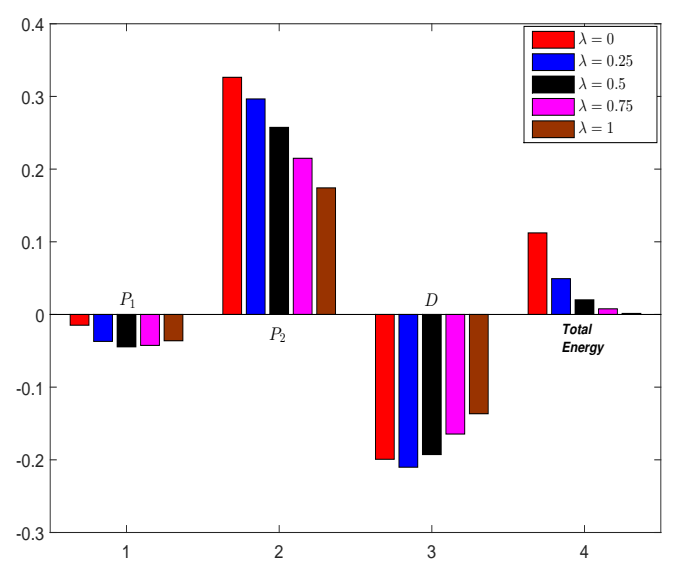

Figure 1. Energy balance of the Ekman boundary layer at $R e=R_{c}+25$ for azimuthal anisotropic roughness (radial grooves). Type I instability mode.

amplified $\left(\alpha_{i}<0\right)$ when energy production outweighs the energy dissipation in the system. Using this formulation it is possible to discuss the effect of roughness on the instabilities of the Type I and Type II modes by calculating the total energy of the system which is the sum of all terms in Eq. (5). If the total energy increases as the roughness parameters are increased, then the modes become unstable. In contrast, a reduced total energy would indicate a stabilization effect.

Underlying our approach to the calculations is a stability code that uses a Chebyshev polynomial discretization method in the wall-normal direction to obtain each term in Eq. (5) for various values of the roughness parameters. Consistent with the recent literature $[4,6,21]$, we use the parameters $\eta$ and $\lambda$ to denote empirical levels of roughness in the radial and azimuthal directions, respectively. The case $\eta>0$ and $\lambda=0$ corresponds to radial anisotropic roughness (concentric grooves); $\lambda>0$ and $\eta=0$ corresponds to azimuthal anisotropic roughness (radial grooves); and $\lambda=\eta>0$ corresponds to isotropic roughness.

\subsection{Results for the Ekman flow}

The energy balance calculation for the Ekman boundary layer is carried out at the location of maximum amplifications of the both Type I and Type II modes at $R e=R_{c}+25$. Here $R_{c}$ is the critical Reynolds number for the onset of the Type I mode of instability for the particular rough surface being considered. Results for various levels of roughness are compared to smooth disk case in Figures 1-3.

Figure 1 shows the energy balance calculation for azimuthal anisotropic roughness (radial grooves). It reveals a stabilization effect on the Type I mode that arises mainly from the large reduction in energy production term $P_{2}$. Note that the energy dissipation of the system, $P_{1}+D$, is largely invariant, leading to a reduction in the total energy of the system with increased roughness of this type. Figure 2 shows a similar stabilizing effect of isotropic roughness on the Type I mode. 


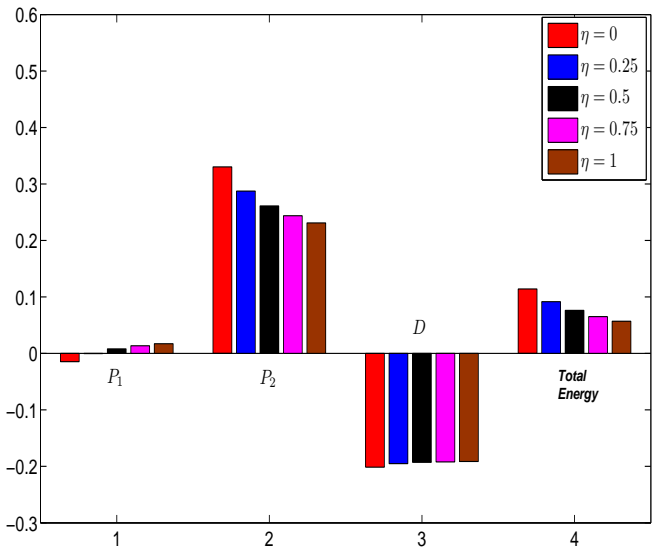

(a) Type I

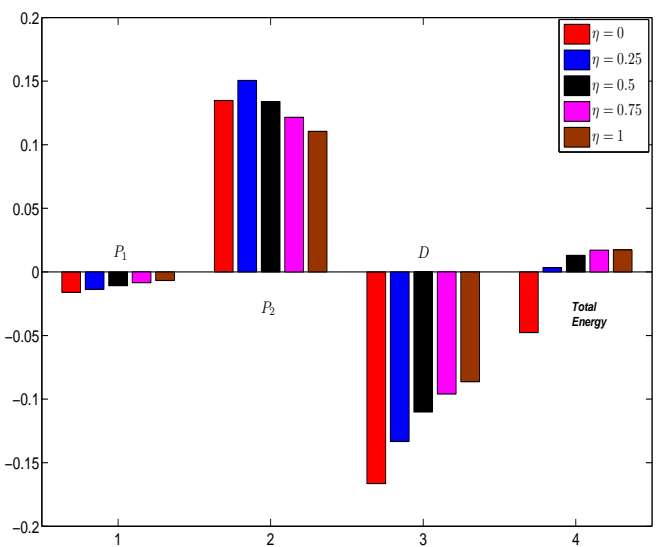

(b) Type II

Figure 3. Energy balance of the Ekman boundary layer at $R e=R_{c}+25$ for radial anisotropic roughness (concentric grooves). (a) Type I instability mode, (b) Type II instability mode.

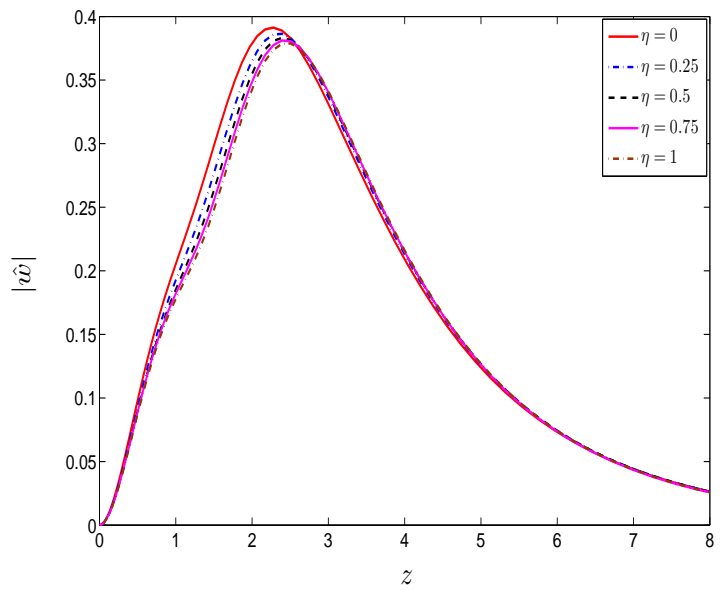

(a) Type I

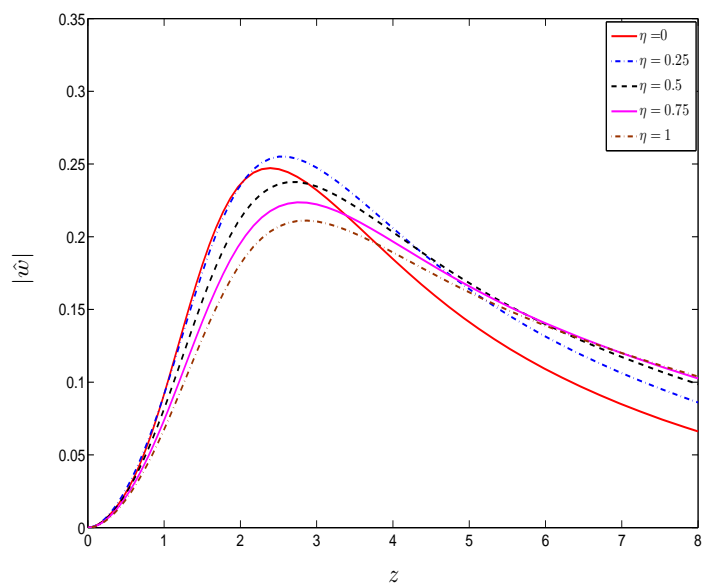

(b) Type II

Figure 4. Profiles of the axial perturbation velocity $w$ of the Ekman layer at $R e=R_{c}+25$ with radial anisotropic roughness (concentric grooves). (a) Type I instability mode, (b) Type II instability mode. 


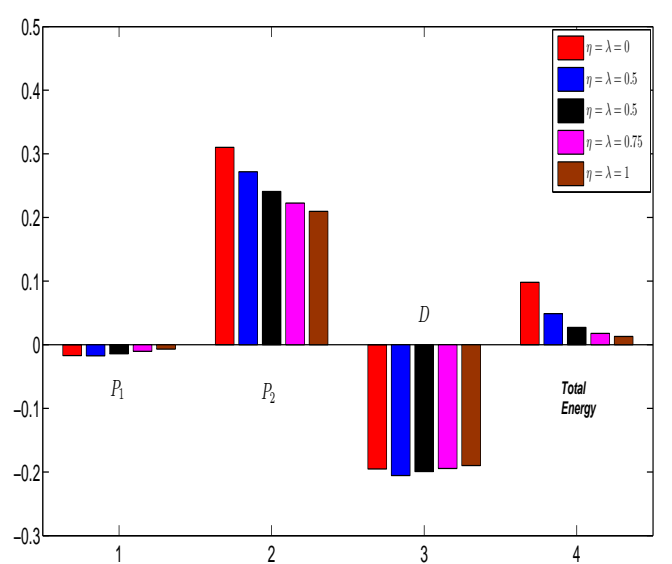

Figure 2. Energy balance of the Ekman boundary layer at $R e=R_{c}+25$ for isotropic roughness. Type I instability mode.

Figure 3(a) demonstrates the major stabilizing effect of radial anisotropic roughness (concentric grooves) on the Type I mode. This is due to a reduction in the energy production term $P_{2}$ and largely invariant viscous stress dissipation $D$. As a result, the total energy of the system decreases. This confirms the stabilization effect of concentric grooves on the Type I mode. In contrast, however, Figure 3(b) presents a major destabilizing effect on the Type II mode. This arises from an increase in $P_{2}$ terms at the points of maximum amplification, together with a slight increase in the viscous dissipation term $D$. Collectively these bring about a increase in the total energy of the system that reveals the destabilization effect of concentric grooves on the Type II mode.

Supportive evidence for the above trends can be obtained from the form of the perturbation velocity profiles, that is, the eigenfunctions. The dominant eigenfunction is the axial perturbation velocity, $w$, which contributes to the dominant energy production term $P_{2}$. Figure 4 shows the magnitude of the $w$-profile for a disk with radial anisotropic roughness. For the Type I mode shown in Figure 4(a), the form of the disturbance profile is largely unchanged as roughness is increased. The reduction in $P_{2}$, however, results from the slight reduction in the amplitude of the profile as roughness is increased. Conversely, Figure 4(b) reveals that the increase of the $P_{2}$ term for the Type II mode is due to an increasing amplitude of this profile with roughness.

\subsection{Results for the Bödewadt boundary layer}

As predicted by Lingwood \& Garrett [23], the very early onset of absolute instability in the Bödewadt flow leads to a "branch exchange" and it is impossible to find the location of maximum amplification of either convective mode. We therefore instead proceed with the energy balance calculations for the Bödewadt boundary layer at fixed $R e=400$ with $n=85$ for the Type I mode and $n=12$ for the Type II mode. These values of $n$ are chosen so as to be outside of the absolutely unstable region at
$\operatorname{Re}=400$.

Figure 5 shows energy terms of Type I and Type II instabilities for $n=85$ and $n=12$, respectively, subject to increasing radial anisotropic roughness. Figure 5(a) reveals that the major stabilizing effect on the Type I mode is from reductions in the energy production by Reynolds stress and the effect of streamline curvature in the boundary layer. The total energy of the system decreases as roughness $(\eta)$ is increased. This is interpreted as evidence of the stabilizing effect of concentric grooves on the Type I mode. Conversely, Figure 5(b) shows a destabilizing effect on the Type II mode that comes from a significant increase in the energy production term $P_{2}$ and subsequent increase in total energy.

Figure 6 demonstrates the destabilizing effect of azimuthal isotropic roughness (radial grooves) on the Type I mode. In particular, increased roughness leads to growth in the energy production terms $P_{2}$ and $G_{1}$. Even though the viscous dissipation is also increased, the net effect is an increase in total energy and a destabilization of the flow.

As shown in Figure 7, increasing the level of isotropic roughness promotes viscous dissipation from the system that causes a net reduction in total energy. This results in a stabilizing effect. In this case, the net energy production in the system is largely invariant.

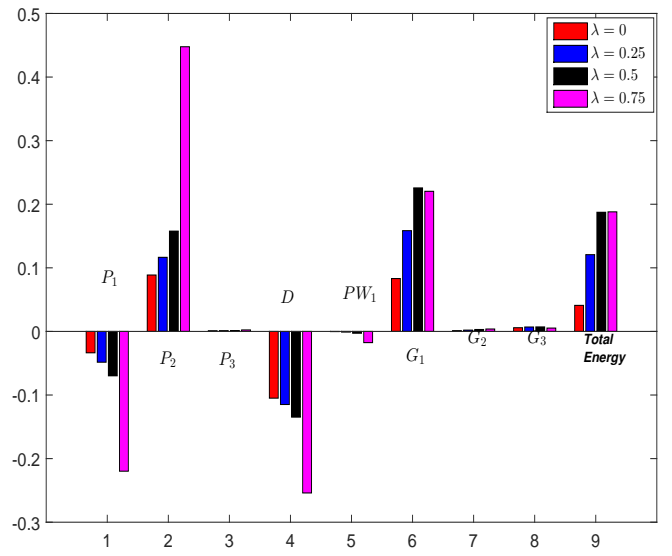

Figure 6. Energy balance of the Bödewadt boundary layer at $R e=400$ for azimuthal anisotropic roughness (radial grooves). Type I instability mode with $n=85$.

Furthermore, Figure 8 shows the magnitude of the $w$ profile for the case of radial anisotropic roughness. For the Type I mode in 8(a), a dramatic reduction in the amplitude of the profile as roughness is increased causes the large reduction in the $P_{2}$ energy production term. That results in a stabilization effect. In contrast, Figure 8(b) reveals that the destabilization of the Type II mode is due to an increase in the amplitude of this profile with roughness. This leads to an increased $P_{2}$ energy production term. 


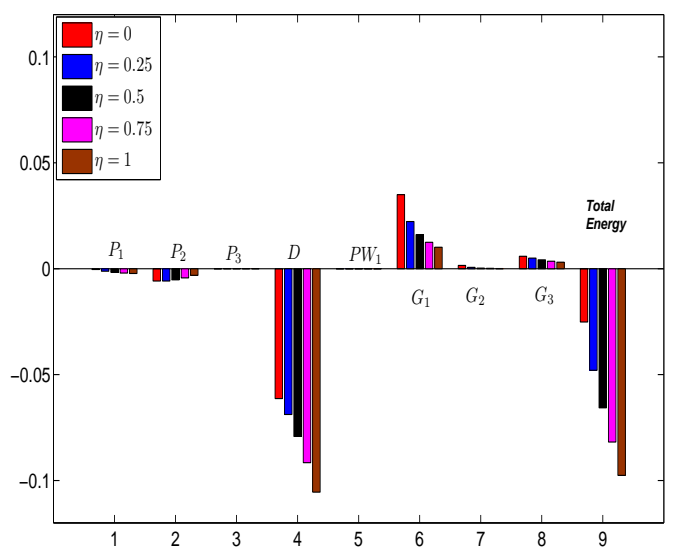

(a) Type I

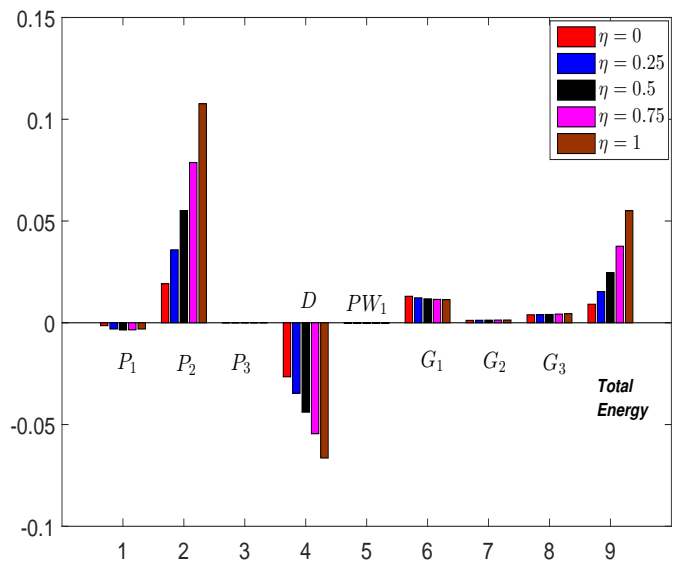

(b) Type II

Figure 5. Energy balance of the Bödewadt boundary layer at $R e=400$ for radial anisotropic roughness (concentric grooves). (a) Type I instability mode with $n=85$, (b) Type II instability mode with $n=12$.

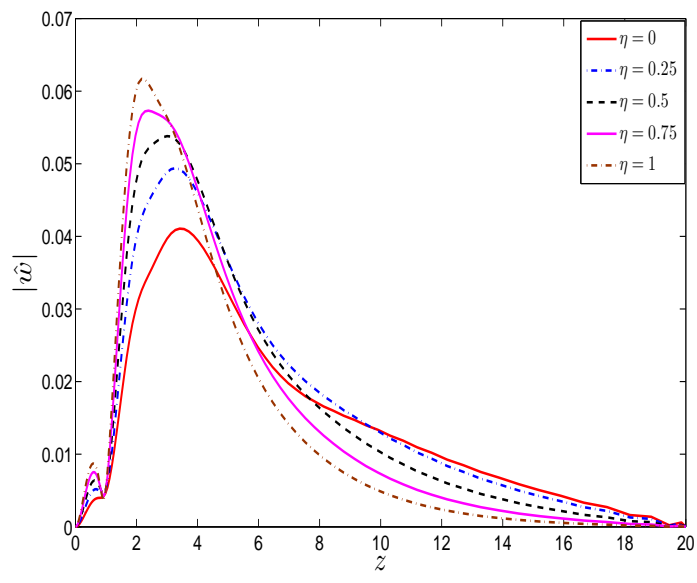

(a) Type I

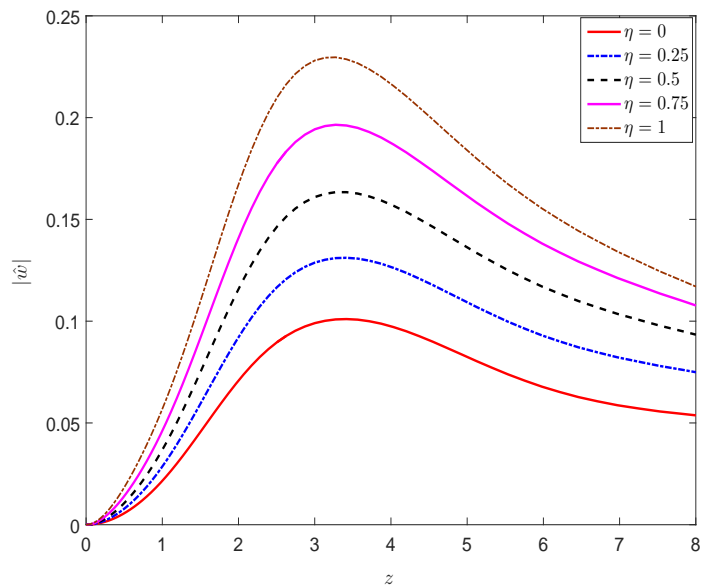

(b) Type II

Figure 8. Profiles of the axial perturbation velocity $w$ of the Bödewadt layer at $R e=400$ with radial anisotropic roughness (concentrically grooved). (a) Type I instability mode, (b) Type II instability mode. 


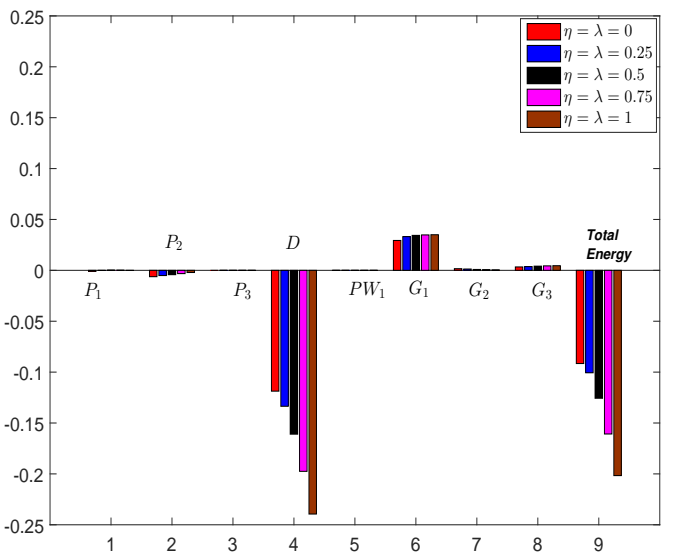

Figure 7. Energy balance of the Bödewadt boundary layer at $R e=400$ for isotropic roughness. Type I instability mode with $n=85$.

\section{CONCLUSION}

This study has been designed to establish the underlying physical mechanisms behind the effects of rough surfaces on the BEK system. The steady flow profiles over the rough disks are modelled using the partial-slip approach of Miklavčič \& Wang [21] without modification. The resulting energy balances are qualitatively consistent with the neutral curve calculations of Alveroglu et al. [6].

Our study has revealed that radially-anisotropic roughness (concentric grooves) and isotropic roughness acts to reduce energy production of the Type I mode for both the Ekman and Bödewadt boundary layers. This is a stabilization effect on the Type I mode. Conversely, azimuthally-anisotropic roughness (radial grooves) is found to have the opposite destabilization effect on the the Type I mode. The energy balance calculation for the Type II mode demonstrates the destabilizing effect of radially-anisotropic roughness for each of the Ekman and Bödewadt boundary layers.

The conclusions arising from this study are easy to interpret: Surface roughness can be used as an effective passive flow-control mechanism for engineering flows of the rotorstator type modelled by the BEK system, and also other cross-flow dominant flows.

\section{ACKNOWLEDGMENTS}

This research used the ALICE High Performance Computing Facility at the University of Leicester. BA wishes to acknowledge financial support from Ministry of National Education, Republic of Turkey. SJG is supported by a Senior Research Fellowship of the Royal Academy of Engineering, funded by the Leverhulme Trust.

\section{REFERENCES}

[1] L. Sirovich and S. Karlsson. Turbulent drag reduction by passive mechanisms. Nature, 388(6644):753-755, 1997.

[2] P. Carpenter. The right sort of roughness. Nature, 388(6644):713-714, 1997.

[3] K. Choi. Fluid dynamics: The rough with the smooth. Nature, 440(7085):754-754, 2006.

[4] A.J. Cooper, J.H. Harris, S.J. Garrett, M. Özkan, and P.J. Thomas. The effect of anisotropic and isotropic roughness on the convective stability of the rotating disk boundary layer. Physics of Fluids (1994-present), 27(1):014107, 2015.

[5] T. von Kármán. Über laminare und turbulente reibung. $Z$. Angew. Math. Mech., 1921.

[6] B. Alveroglu, A. Segalini, and S.J. Garrett. The effect of surface roughness on the convective instability of the bek family of boundary-layer flows. European Journal of Mechanics-B/Fluids, 2015.

[7] U.T. Bödewadt. Die drehströmung über festem grunde. ZAMM-Journal of Applied Mathematics and Mechanics/Zeitschrift für Angewandte Mathematik und Mechanik, 20(5):241-253, 1940 .

[8] V. W. Ekman. On the influence of the Earth's rotation on ocean currents. Ark. Mat. Astron. Fys., 2:1-53, 1905.

[9] Ö. Savas. Stability of Bödewadt flow. Journal of Fluid Mechanics, 183:77-94, 1987.

[10] J.M. Lopez. Flow between a stationary and a rotating disk shrouded by a co-rotating cylinder. Physics of Fluids (1994-present), 8(10):2605-2613, 1996.

[11] R.J. Lingwood and P.H. Alfredsson. Experimental study of the stability of the Bödewadt layer. Springer, 2000.

[12] L. Schouveiler, P. Le Gal, and M.P. Chauve. Instabilities of the flow between a rotating and a stationary disk. Journal of Fluid Mechanics, 443:329-350, 2001.

[13] A. Cros, E. Floriani, P. Le Gal, and R. Lima. Transition to turbulence of the Batchelor flow in a rotor/stator device. European Journal of Mechanics-B/Fluids, 24(4):409-424, 2005.

[14] N.A. Culverhouse. The hydrodynamic stability of crossflow vortices in the Bödewadt boundary layer. $\mathrm{PhD}$ thesis, University of Birmingham, 2009.

[15] P.R. Tatro and E.L. Mollo-Christensen. Experiments on Ekman layer instability. Journal of Fluid Mechanics, 28(03):531-543, 1967.

[16] A.J. Faller. An experimental study of the instability of the laminar Ekman boundary layer. Journal of Fluid Mechanics, 15(04):560-576, 1963. 
[17] R.J. Lingwood. Absolute instability of the ekman layer and related rotating flows. Journal of Fluid Mechanics, 331:405-428, 1997.

[18] B.I. Fedorov, G.Z. Plavnik, I.V. Prokhorov, and L.G. Zhukhovitskii. Transitional flow conditions on a rotating disk. Journal of Engineering Physics and Thermophysics, 31(6):1448-1453, 1976.

[19] T.C. Corke and K.F. Knasiak. Stationary travelling crossflow mode interactions on a rotating disk. Journal of Fluid Mechanics, 355:285-315, 1998.

[20] R.J. Lingwood and P.H. Alfredsson. Instabilities of the von Kármán boundary layer. Applied Mechanics Reviews, 67(3):030803, 2015.

[21] M. Miklavčič and C.Y. Wang. The flow due to a rough rotating disk. Zeitschrift für angewandte Mathematik und Physik ZAMP, 55(2):235-246, 2004.

[22] M. S. Yoon, J. M. Hyun, and Jun Sang P. Flow and heat transfer over a rotating disk with surface roughness. International journal of heat and fluid flow, 28(2):262267, 2007.

[23] R.J. Lingwood and S.J. Garrett. The effects of surface mass flux on the instability of the bek system of rotating boundary-layer flows. European Journal of MechanicsB/Fluids, 30(3):299-310, 2011.

[24] A.J. Cooper and P.W. Carpenter. The stability of rotatingdisc boundary-layer flow over a compliant wall. part 1. type I and II instabilities. Journal of Fluid Mechanics, 350:231-259, 1997. 